\title{
EARLY DIAGNOSIS OF SPINAL TUBERCULOSIS BY MRI
}

S. S. DESAI

From the University of Bombay, India

MRI was performed at three centres in Bombay on 24 patients clinically suspected of tuberculosis of the spine but with normal radiographs. There were 11 males and 13 females and their average age was 24 years $(11$ to 60$) .{ }^{99} \mathrm{~m} T$ bone scans were done in 16 patients before MRI. Eleven patients had the diagnosis confirmed by biopsy and the remainder all responded rapidly to specific antituberculous chemotherapy.

On T1-weighted images there was a decrease in signal intensity of the involved bone and soft tissues; on T2-weighted images there was increased signal intensity. Depending on the stage of the disease, three different patterns of infection were revealed: osteitis, osteitis with an abscess, and osteitis with or without an abscess plus discitis. The anatomical pattern of involvement, particularly of the soft tissues and the discs, is specific for tuberculous disease. The ability of MRI to detect tuberculosis of the spine earlier than other techniques could reduce bone destruction and deformity and diminish the need for surgical intervention.

Despite the specificity of the patterns revealed by MRI, biopsy is recommended during the stage of osteitis to confirm the diagnosis.

J Bone Joint Surg [Br] 1994; 76-B:863-9.

Received 3 February 1994; Accepted 20 April 1994

Bone and joint tuberculosis continues to be a major problem in most developing countries and there has recently been evidence of resurgence in the western world (Watson 1993). The several MRC trials (MRC Working Party on Tuberculosis of the Spine 1978, 1982, 1985, $1986,1993)$ established that drug therapy is the mainstay of treatment for tuberculosis of the spine and that surgery is needed only when there is gross destruction, deformity or associated neurological deficit not responding to chemotherapy. One of the causes of such destruction and deformity is delay in diagnosis. More than $50 \%$ of the vertebra has to be destroyed before a lesion can be seen

S. S. Desai, MCh, MS, D Orth, DNB, Consultant Orthopaedic Surgeon c/303, Swati Apartments, Juhu Lane, Andheri (West), Bombay-400058, India.

C1994 British Editorial Society of Bone and Joint Surgery

$0301-620 \mathrm{X} / 94 / 6872 \$ 2.00$ on a plain radiograph, a process which takes nearly six months (Lifeso, Weaver and Harder 1985).

One of the aims of all surgical advances should be to reduce, if possible, the need for surgical intervention. The purpose of this study is to define the MRI appearance of tuberculosis of the spine, which has hitherto not been well described in the literature (Modic et al 1985; DeRoos et al 1986), in an attempt to allow diagnosis of the disease at an earlier stage than is possible by conventional techniques.

\section{PATIENTS AND METHODS}

Between 1989 and 1991, 24 patients with suspected tuberculosis of the spine underwent MRI at one of three hospitals in Bombay. There were 11 males and 13 females with an average age of 24.6 years (11 to 60 ). There were only three children in this series. In all cases there was a strong clinical suspicion of spinal tuberculosis based on the history and symptoms and signs. The diagnosis was eventually established by histological examination in 11 patients and by response to specific chemotherapy in the others.

All the patients had undergone routine laboratory tests. A raised ESR was not thought to be significant in the diagnosis of tuberculosis, but was considered to be a useful measure of response to treatment.

All patients had had anteroposterior and lateral radiographs of the spine and chest. The radiographs were used to measure a decrease in the height of the intervertebral disc and to detect erosion of the vertebral end-plate, lytic lesions in the vertebrae and paravertebral abscesses.

Radioisotope bone scanning was undertaken before MRI in 16 patients. In the remaining eight, seven of whom had a neurological deficit and one a clinically detectable paraspinal abscess, no bone scan was performed. Scanning was by a Siemens rota camera with a low-energy, high-resolution collimator (Iselin, New Jersey). Twenty millicuries of ${ }^{99 \mathrm{~m}} \mathrm{Tc}$ hydroxydiphosphonate were given intravenously and static whole-body images were obtained three hours after the injection. Markedly increased activity in the suspected area of the spine was considered sufficient reason for further investigation by MRI.

MRI was performed with Hitachi MRP-20 or MRmax (0.5 Tesla, GE Medical, Waukesha, Milwaukee) superconducting units at three imaging centres in Bombay. 
Table I. MRI findings in 17 patients with vertebral-body involvement

\begin{tabular}{|c|c|c|c|c|c|c|c|}
\hline \multirow{2}{*}{$\begin{array}{l}\text { MRI } \\
\text { pattern }\end{array}$} & \multirow[b]{2}{*}{ Number } & \multicolumn{2}{|c|}{ Vertebral body } & \multicolumn{2}{|l|}{ Disc } & \multicolumn{2}{|l|}{ Abscess } \\
\hline & & T1-weighted & T2-weighted & T1-weighted & T2-weighted & T1-weighted & T2-weighted \\
\hline Osteitis & 2 & $\mathrm{D}^{*}$ & I† & $\mathrm{N} \ddagger$ & $\mathbf{N}$ & A§ & A \\
\hline $\begin{array}{l}\text { Osteitis } \\
\text { and abscess }\end{array}$ & 7 & D & I & $\mathbf{N}$ & $\mathbf{N}$ & D & I \\
\hline $\begin{array}{l}\text { Osteitis, } \\
\text { abscess } \\
\text { and discitis }\end{array}$ & 8 & D & I & D & I & D & I \\
\hline
\end{tabular}

* decreased signal intensity in comparison with the normal vertebral disc

$\dagger$ increased signal intensity

$\ddagger$ normal signal intensity

$\S$ absent

All patients were studied in the coronal, sagittal and axial planes using T1-weighted (TR $600 \mathrm{~ms}$; TE $25 \mathrm{~ms}$ ) and T2-weighted (TR $2600 \mathrm{~ms}$; TE $90 \mathrm{~ms}$ ) spin-echo images. Nine patients had gradient-echo sequences. Gadolinium diethylene triaminepenta-acetic acid (DTPA) contrast was not used.

MRI was performed to obtain the diagnosis and also for preoperative planning in those patients who required surgical treatment. The images were examined for configuration and intensity of the signal from the adjacent vertebral bodies and the intervening disc. The disc height was compared with the height of the discs above and below. Soft-tissue changes in front or at the side of the vertebral body were identified as pre- or paravertebral abscess or granuloma. Intraspinal extension was recorded and correlated with neurological deficit. The signal intensity in the normal vertebral bodies and discs in the field of view served for comparison. Two patients had repeat MRI one year after antituberculous chemotherapy.

\section{RESULTS}

Clinical features. All but one of the patients presented with back pain and spasm which had not responded to conservative treatment; the remaining patient had symptoms of radiculopathy with restricted straight-leg raising. Only one patient had a clinically detectable paraspinal abscess, palpable posteriorly over the cervical spine. None had any type of spinal deformity. Of the four patients with general symptoms of tuberculosis, two had associated pulmonary disease and two had tuberculous cervical lymphadenopathy. Of the seven patients with neurological deficit, two had paraparesis, two had paraparesis with bladder and bowel involvement, two had cauda equina syndrome and one had S1 root entrapment with loss of the ankle reflex and hypoaesthesia along the lateral border of the foot.

The lesion was cervical in eight patients, thoracic in 13 , and lumbar in seven. The mean duration of symptoms was 5.2 months (2.4 to 8.4 ).

Radiography. Six patients had pulmonary lesions visible on the radiographs.
Table II. Duration of symptoms related to the three MRI patterns shown in Table I

\begin{tabular}{ll}
\hline MRI pattern & $\begin{array}{l}\text { Duration of symptoms } \\
\text { (mth; range) }\end{array}$ \\
\hline Osteitis & $3.2(2.4$ to 4.1$)$ \\
Osteitis and abscess & $5.1(4.0$ to 6.2$)$ \\
$\begin{array}{l}\text { Osteitis, abscess } \\
\text { and discitis }\end{array}$ & $7.2(6.1$ to 8.4$)$ \\
\hline
\end{tabular}

All the spinal radiographs were normal showing no osseous or disc changes and no abscesses.

Scintigraphy. Fourteen of the 16 patients who had isotope scans had positive results and two were negative. The latter were found to have tuberculous lesions of the posterior elements, the vertebral body being normal. The rest of the skeleton was normal in all the patients.

Magnetic resonance imaging. The results showed involvement in 23 patients ( 37 vertebral bodies); $12 \mathrm{had}$ one vertebra involved, eight had two adjacent vertebrae involved and three had three or more vertebrae affected. Twenty-two patients had an abscess. Only one of these was detected clinically, a posterior paraspinal abscess with no bone involvement.

In all cases the involved tissues showed a decrease in signal intensity on T1-weighted images and an increase in intensity on T2-weighted images. Three different patterns were identified, depending on involvement of the discs and the presence or absence of an abscess (Tables I and II). Those affecting the $\mathrm{C} 1-\mathrm{C} 2$ vertebrae and those involving only the posterior elements are shown separately in Tables III and IV.

In the 17 patients with typical vertebral body involvement, the lesion was anteroinferior in six, paradiscal or metaphyseal in six, central in two and posterior in three. Fifteen patients had an abscess. Two patients had neither an abscess nor disc involvement, only vertebral body involvement (Fig. 1). They had repeat MRI after antituberculous chemotherapy which showed reversal of the changes in signal intensity.

The disc was involved in eight of these 17 patients. Of the remaining nine, without disc involvement, seven 


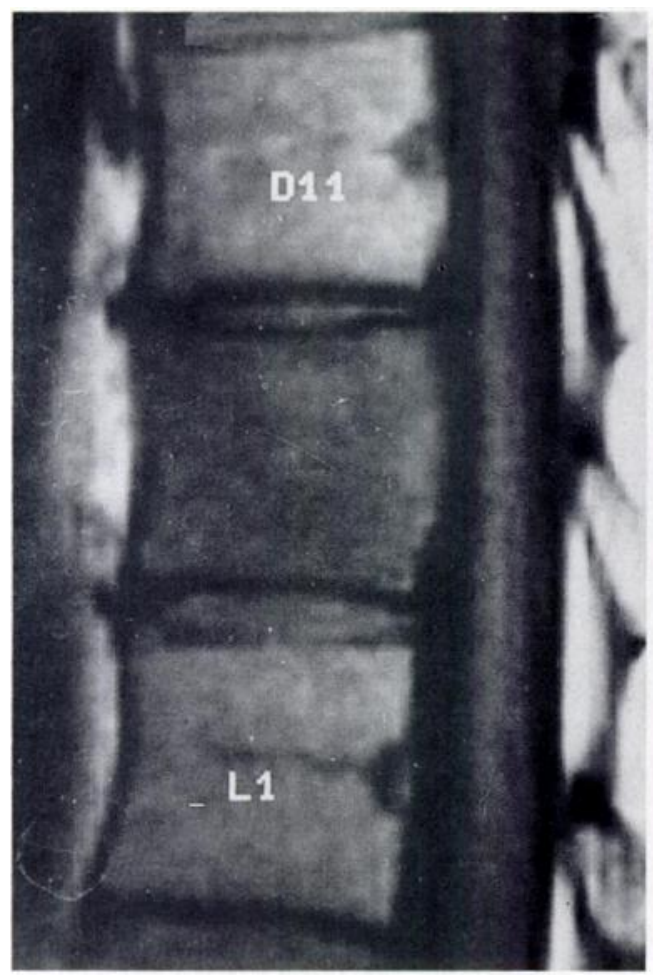

Fig. 1a

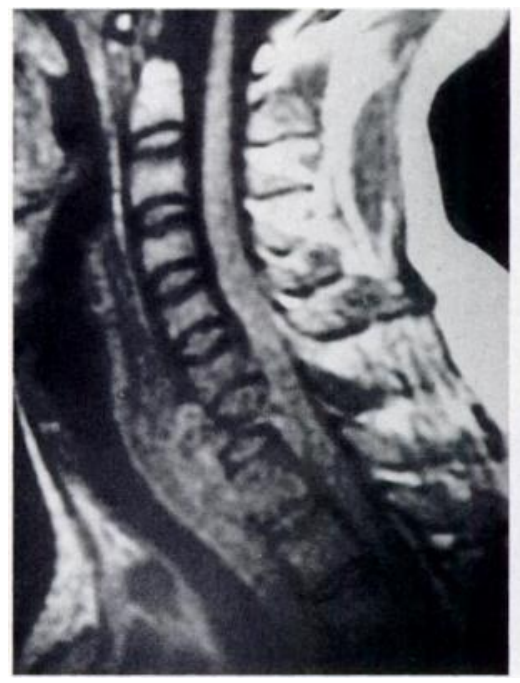

Fig. 2a

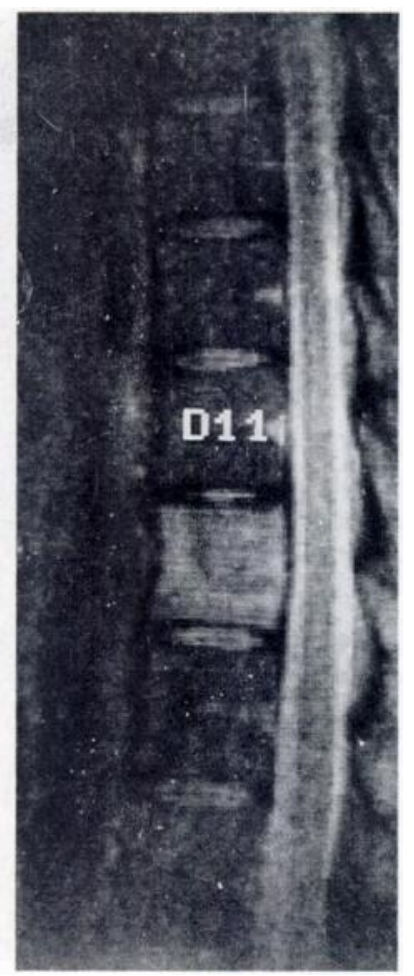

Fig. 1b

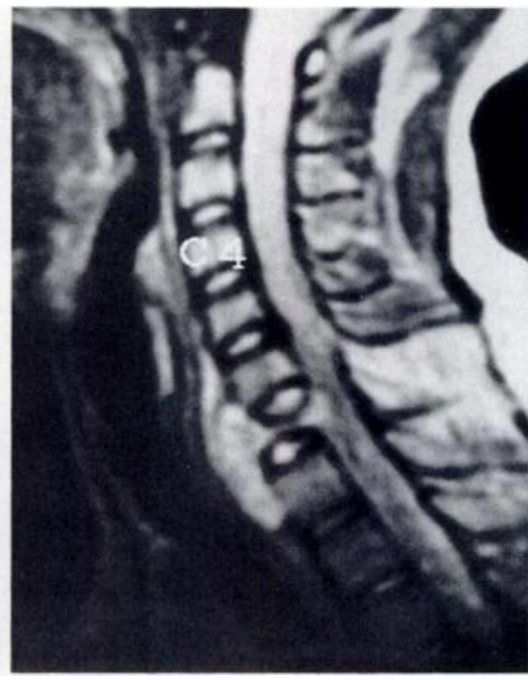

Fig. 2b
Osteitis. Figure 1a - The T1-weighted MR image shows decreased signal intensity of the D12 vertebral body. The discs above and below are normal and there is no abscess. Figure 1b - The T2-weighted MR image shows increased signal intensity of D12.

\begin{abstract}
Osteitis and an abscess. Figure $2 \mathrm{a}-$ The $\mathrm{T} 1$ weighted MR image shows decreased signal intensity of the $\mathrm{C} 7$ vertebral body. The prevertebral abscess shows similar signal changes, but the disc height and signal are normal. Figure $2 \mathrm{~b}-$ The T2-weighted image shows increased signal intensity of $\mathrm{C} 7$ and similar signal changes in the prevertebral and intraspinal abscess. There is a ballooned appearance of the otherwise normal discs above and below.
\end{abstract}

had a paraspinal abscess. All the abscesses had smooth margins and extended under the anterior longitudinal ligament (Fig. 2). In two of the nine the disc appeared ballooned but there was no abnormality in its signal intensity (Fig. 2). This appearance is probably due to weakening of the vertebral end-plate.

All the six patients with compression of the spinal cord or cauda equina had an epidural abscess or granuloma occupying more than $50 \%$ of the spinal canal. Two of these six were already improving with antituberculous chemotherapy, and did not undergo decompression. They both had disc involvement shown on MRI.

$C 1$ and $C 2$ vertebrae. Two patients had a lesion in $\mathrm{C} 1$, one had a C2 lesion and one had lesions in $\mathrm{C} 1$ and $\mathrm{C} 2$ (Fig. 3). In all four the lateral mass showed decreased signal intensity on T1-weighted images and increased signal intensity on T2-weighted images and all four had paravertebral abscesses showing similar signal changes. None had any intraspinal extension; this correlates with the absence of neurological deficits. Two of these patients had enlarged cervical lymph nodes within the carotid sheath, displaying decreased signal intensity on T1weighted images and increased intensity on T2-weighted images. Both were proved to be tuberculous on biopsy of the lymph nodes.

Posterior elements. One patient had involvement of the 


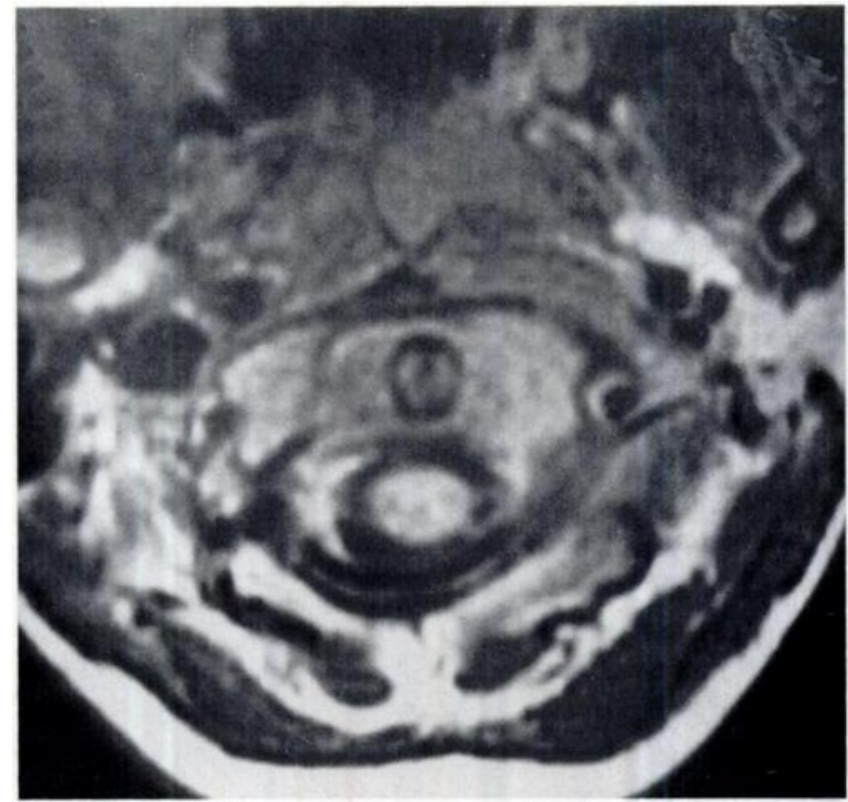

Fig. 3a

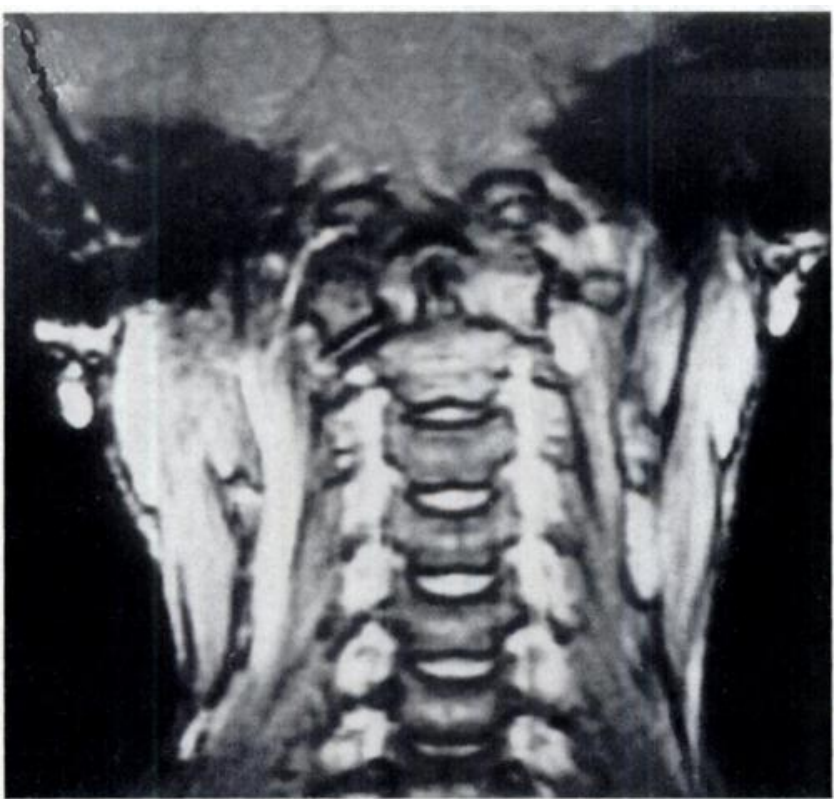

Fig. 3c

lamina and facet joint of L5 and S1, with an abscess in the lateral recess encroaching on the S1 nerve root. One had involvement of the spinous process of L3 with a large posterior paraspinal abscess extending from L2 to L4 (Fig. 4). Both these patients had normal signal intensity from the vertebral body and disc on T1- and T2-weighted images.

Treatment and review. All the patients were treated with rifampicin (15 mg/kg body-weight) and isoniazid $(6 \mathrm{mg} /$ $\mathrm{kg}$ body-weight) for nine months.

Four patients had spinal decompression for progressive neurological deficit. The patient with symptoms of radiculopathy had a laminectomy and removal of a tuberculous granuloma pressing on the nerve root in the

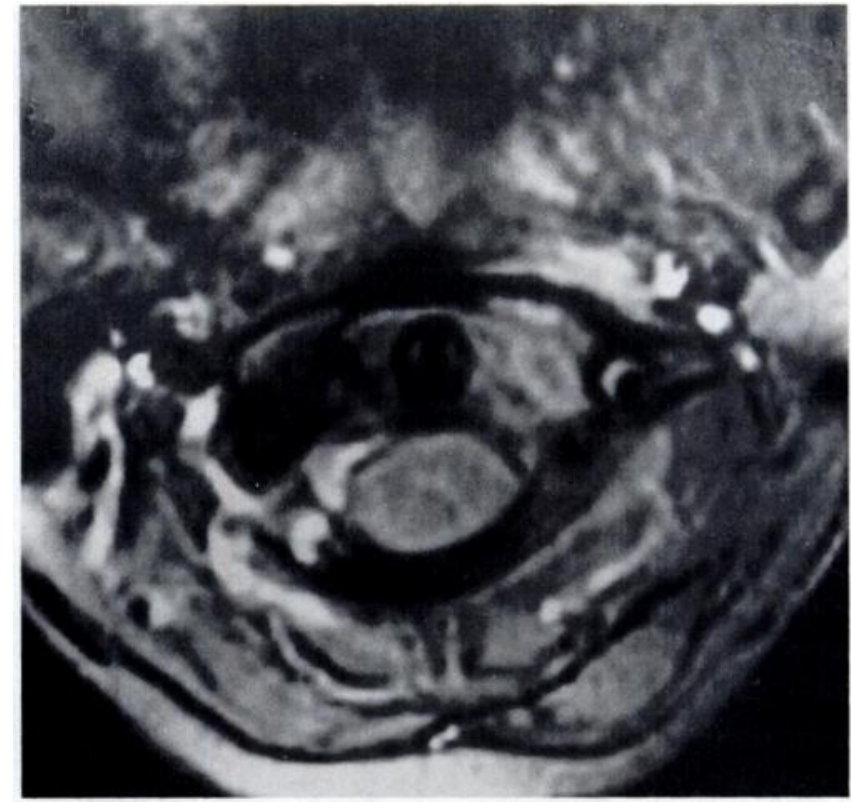

Fig. 3b
Osteitis of lateral mass of $\mathrm{Cl}$ vertebra and an abscess. Figure $3 \mathrm{a}$ - The $\mathrm{T} 1$ weighted axial MR image of the $\mathrm{Cl}$ vertebra shows decreased signal intensity of the left lateral mass in comparison with the right. The left paravertebral abscess shows similar signal changes. Figure $3 b-$ The T2weighted axial MR image shows increased signal intensity of the left lateral mass. The left paravertebral abscess shows similar signal changes. Figure $3 c-$ The T2-weighted coronal MR image shows increased signal intensity of the left lateral mass, and destruction of the articulation with C2. lateral recess. Two patients had needle biopsy of the vertebra, two had lymph-node biopsy from the cervical region, and one had a pleural biopsy, all of which showed histological evidence of tuberculosis. The patient with a clinically detectable, cervical paraspinal abscess underwent drainage and curettage of the abscess wall, and histological examination confirmed tuberculosis.

The 13 patients who did not have a surgical procedure all showed a clinical and haematological response to antituberculous treatment. Two had repeat MRI after chemotherapy which showed cure. All the patients were asymptomatic six months after completion of treatment and there was complete neurological recovery in them all at one year. No patient developed any spinal deformity. 


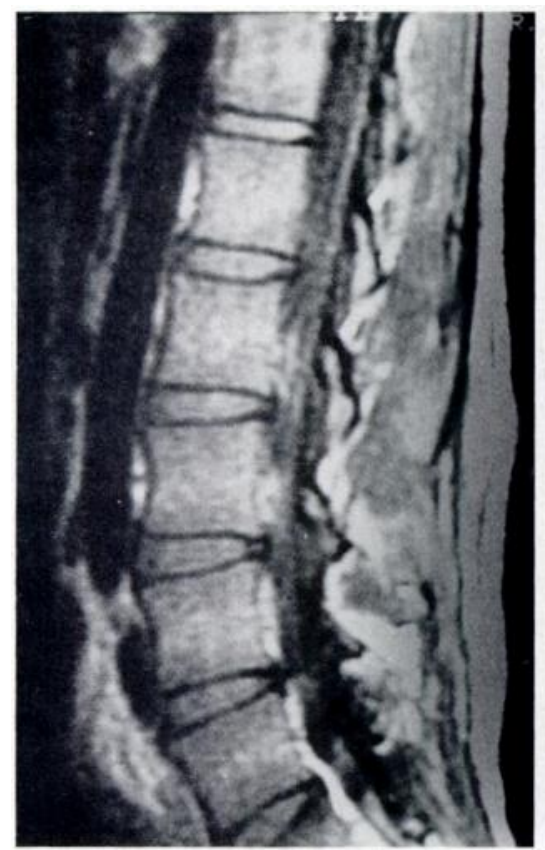

Fig. 4a

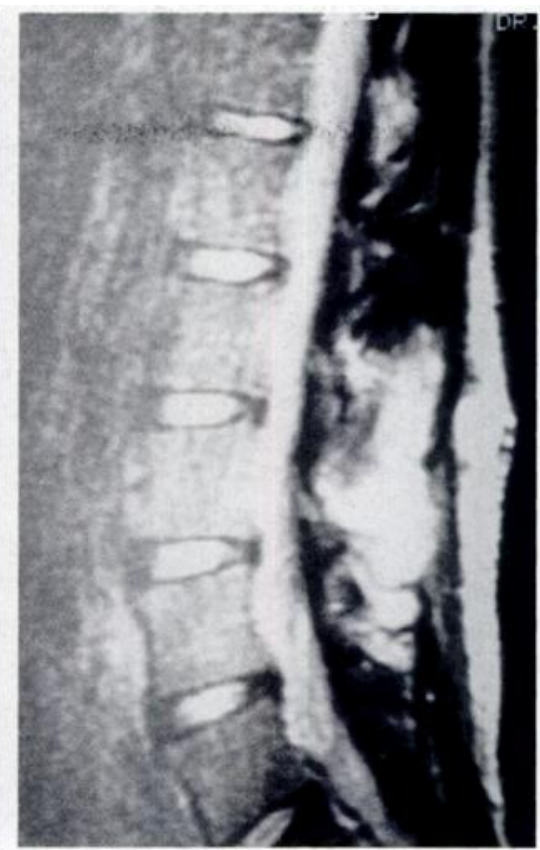

Fig. $4 b$
Posterior element lesion. The T1-weighted (left) and T2-weighted (right) MR images show normal signal intensity of the vertebrae and discs. The T2-weighted MR image (right) shows increased signal from the soft tissues posteriorly and a paraspinal abscess.

\section{DISCUSSION}

Most of the patients in this study were under 40 years of age. Diagnosis is easier in this age group because diseases which may mimic tuberculosis are uncommon.

The three patients aged more than 50 years all had disc involvement and an abscess shown by MRI making the diagnosis secure in correlation with the clinical findings.

Modic et al (1985) reported that scintigraphy was as sensitive as MRI in the diagnosis of vertebral osteomyelitis. In our study the isotope scan was positive in $87.5 \%$ of cases, but it did not allow us to make a definite diagnosis of tuberculosis. The method is less sensitive than MRI for the detection of soft-tissue infection (Beltran et al 1988). Lifeso et al (1985) found ${ }^{99 \mathrm{~m}} \mathrm{Tc}$ bone scanning to be negative in $35 \%$ of cases with radiographically demonstrable lesions. In our series the two false-negative results were in those cases with involvement of only the neural arch. We have found that scintigraphy is a sensitive method which can be used as a relatively inexpensive investigation to decide which patients are likely to benefit from MRI, particularly at the stage when the radiographs are normal.

The ability of MRI to detect the disease early has allowed confirmation of what Massart and Ducroquet (1926) stated nearly seventy years ago: "that in Pott's disease the abscess remains 'hidden' but may be detected by X-ray and its presence is absolute evidence of Pott's disease'. It has also been possible actually to document the pathogenesis of tuberculosis of the spine as postulated in the literature (Butler 1935). The two patients in whom vertebral body involvement only was seen, with no abnormality of the adjacent disc and soft tissues, are a clear indication that the infection starts in the bone. These two patients had the shortest duration of symptoms and had MRI three months after their onset. About half the patients had vertebral body involvement with an abscess but a normal disc at an average duration of 5.1 months, demonstrating that the formation and spread of the abscess occur next in the progression of the disease. The disc is only secondarily affected and at a later stage. The ballooning of discs with a normal MRI suggests that the narrowing of the disc seen on radiographs is due to its herniation through the weakened end-plate and is not the result of disease of the disc itself (Weaver and Lifeso 1984). The site of formation of an abscess, whether prevertebral, paravertebral or intraspinal, did not correlate with the site of the initial lesion in the vertebral body. In the later stages of the disease all these changes may be present simultaneously.

We detected infection of bone and soft tissue on MRI by a decrease in signal intensity on T1-weighted images and an increase on T2-weighted images, as have others (Modic et al 1985; DeRoos et al 1986; Smith et al 1989). These changes are not in themselves specific for infection but only when they are associated with evidence of a paravertebral or prevertebral abscess or disc involvement. Our study has proved correct what was stated nearly a hundred years ago by Nichols (1898): "In a considerable proportion of cases of tubercular disease of the spine no abscess is recognised during life, but in cases seen at autopsy an abscess is almost invariably found, although it may be small in size".

MRI demonstrated best the soft-tissue lesion, which is not imaged as well by any other method (Paushter, Modic and Masaryk 1985). Twenty-two of our 24 patients had an abscess and the spread of pus was well demon- 
Table III. MRI findings in six patients with atypical vertebral involvement

\begin{tabular}{|c|c|c|c|c|c|c|c|}
\hline \multirow{2}{*}{$\begin{array}{l}\text { MRI } \\
\text { pattern }\end{array}$} & \multirow[b]{2}{*}{ Number } & \multicolumn{2}{|c|}{ Vertebral body } & \multicolumn{2}{|l|}{ Disc } & \multicolumn{2}{|l|}{ Abscess } \\
\hline & & T1-weighted & T2-weighted & T1-weighted & T2-weighted & T1-weighted & T2-weighted \\
\hline $\begin{array}{l}\mathrm{C} 1-\mathrm{C} 2 \\
\text { (lateral mass) }\end{array}$ & 4 & $D^{*}$ & It & - & - & D & I \\
\hline $\begin{array}{l}\text { Posterior } \\
\text { element } \\
\text { (vertebral } \\
\text { body normal) }\end{array}$ & 2 & D & I & $\mathrm{N} \ddagger$ & $\mathbf{N}$ & D & I \\
\hline
\end{tabular}

* decreased signal intensity in comparison with the normal vertebral disc

$\dagger$ increased signal intensity

$\ddagger$ normal signal intensity

strated by MRI, particularly under the anterior longitudinal ligament resulting in a notably smooth margin. The pattern of spread of the abscess can be used to differentiate between tuberculous and pyogenic infections on MRI. The smooth margin of a cold abscess, due to its subligamentous spread, contrasts with the irregular margin of a pyogenic abscess which destroys the paraspinal ligament and invades the para-aortic or precaval space (Kang et al 1992). According to Kang et al (1992) postgadolinium-DTPA MRI shows either an irregular thick or a uniform thin rim enhancement suggesting either caseation necrosis or a cold abscess in tuberculosis; pyogenic abscesses show diffuse enhancement. We do not suggest that it is possible to differentiate other chronic granulomatous conditions from tuberculous infection exclusively on MRI; clinical correlation is always necessary. In the absence of an abscess or involvement of a disc on MRI, a biopsy is always recommended and it may be considered obligatory in every case in countries in which tuberculosis is not common. It may be unwise to extrapolate the suggestions based on this study to areas where the disease is rarely met with.

Seddon, in 1935, first described 'spinal tumour syndrome' as a form of tuberculosis of the spine in which neurological symptoms and signs precede radiological changes. The diagnosis of tuberculosis in these patients was established only in retrospect, after obtaining a biopsy while decompressing the cord. In our patients MRI detected the tuberculous lesion in bone in all seven patients with neurological deficit and normal radiographs. All of them had bone lesions which were visible by MRI but not by radiography (Fig. 5). Patients with so-called 'spinal tumour syndrome' probably had a bone lesion as well as an epidural granuloma or abscess, but it was undetectable by the imaging techniques then available. A larger MRI study is required to show whether extradural, extraosseous disease really exists (Rao et al 1965). With our enhanced ability to diagnose these patients as

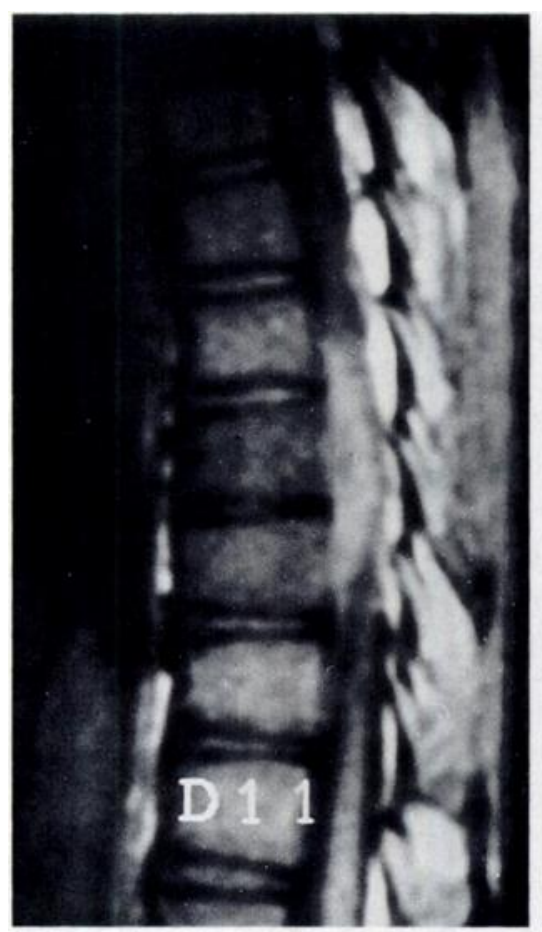

Fig. 5a

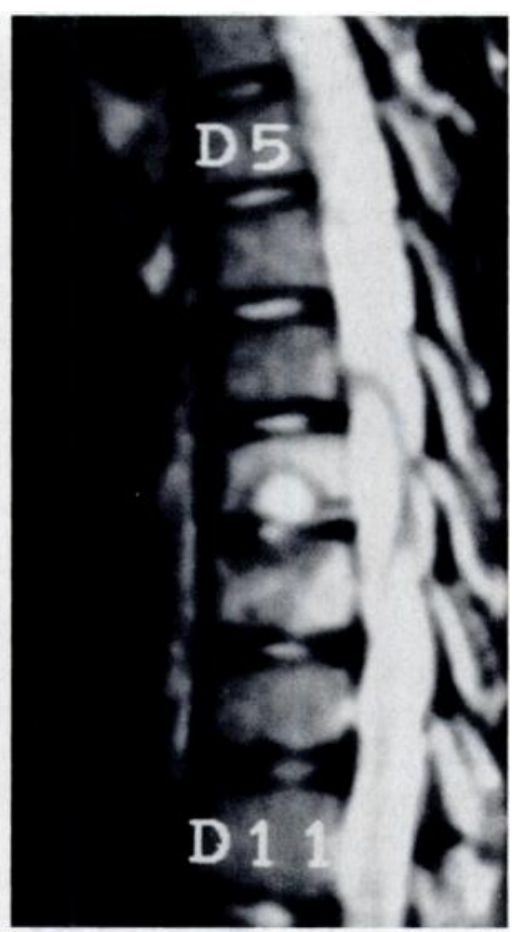

Fig. $5 b$
'Spinal tumour syndrome'. Figure $5 \mathrm{a}-$ The T1-weighted MR image shows decreased signal intensity of T8 and T9 vertebral bodies with a large intraspinal abscess. Figure $5 b-$ The T2-weighted MR image shows increased signal intensity of $\mathrm{T} 8$ and $\mathrm{T} 9$ vertebral bodies with a large intraspinal abscess. 
Table IV. Duration of symptoms related to the two MRI patterns shown in Table III

\begin{tabular}{ll}
\hline MRI pattern & $\begin{array}{l}\text { Duration of symptoms } \\
\text { (mth; range) }\end{array}$ \\
\hline $\begin{array}{l}\text { C1-C2 } \\
\text { (lateral mass) }\end{array}$ & $5.6(3.8$ to 7.4$)$ \\
$\begin{array}{l}\text { Posterior element } \\
\text { (vertebral body normal) }\end{array}$ & $5(4.1$ to 5.9$)$ \\
\hline
\end{tabular}

tuberculous when they present with symptoms of a spinal tumour, the need for surgical intervention has been greatly reduced.

In those regions of the spine in which visualisation of lesions by radiography is difficult, such as in the occipitocervical and cervicothoracic junctions and in the posterior elements, MRI has proved very valuable. The MRI features of occipitocervical and atlantoaxial tuberculosis have not been described before in the English literature. The early detection of the disease in a potentially life-threatening area allowed its rapid cure by chemotherapy. Neural arch tuberculosis is rare and diagnosis is often delayed leading to irreversible neurological sequelae in a high proportion of cases (Naim-Ur-Rahman 1980). These lesions can also be detected early by MRI and a posterior rather than the usual anterior or anterolateral approach may be selected if decompression is needed.

The use of MRI in preoperative planning has been described before (Bell et al 1990). The method best shows the extent of the lesion and the precise level of cord compression responsible for any neurological deficit and helps in deciding on the surgical approach in cases that need decompression.

Conclusion. MRI is more sensitive than radiography and more specific than scintigraphy in the diagnosis of spinal tuberculosis. It is the anatomical pattern revealed by MRI, particularly the soft-tissue and disc involvement, that gives specificity. The possibility which it presents of the diagnosis of tuberculosis of the spine four to six months earlier than by conventional methods offers great benefits. It must be emphasised, however, that MRI must be correlated with clinical examination and that it has its limitations. The method is costly and its power of differentiation between tuberculosis and other chronic infections needs further evaluation. The role of gadolinium-DTPA contrast also remains to be explored.

The author wishes to thank the staff of the MRI centres in Bombay for their co-operation in collecting the cases.

No benefits in any form have been received or will be received from a commercial party related directly or indirectly to the subject of this article.

\section{REFERENCES}

Bell GR, Stearns KL, Bonutti PM, Boumphrey FR. MRI diagnosis of tuberculous vertebral osteomyelitis. Spine 1990; 15:462-5.

Beltran J, McGhee RB, Shaffer PB, et al. Experimental infections of the musculoskeletal system: evaluation with MR imaging and Tc-99M MDP and Ga-67 scintigraphy. Radiology 1988; 167:167-72.

Butler RW. Paraplegia in Pott's disease with special reference to pathology and etiology. Br J Surg 1935; 22:738-68.

DeRoos A, Van Persijn Van Meerten EL, Bloem JL, Bluemm RG. MRI of tuberculous spondylitis. AJR 1986; 147:79-82.

Kang HS, Kim SM, Chang KH, Kim CW, Han MC. Differentiation of tuberculous and pyogenic spondylitis: value of MR imaging. Proc 78th Scientific Assembly and Annual Meeting of the Radiology Society of North America, 1992.

Lifeso RM, Weaver P, Harder EH. Tuberculous spondylitis in adults. $J$ Bone Joint Surg [Am] 1985; 67-A:1405-13.

Massart R, Ducroquet R. Radiographic exploration of abscesses in Pott's disease. Arch Franco-belges Chir 1926; 29:181-201.

Modic MT, Feiglin DH, Piraino DW, et al. Vertebral osteomyelitis: assessment using MR. Radiology 1985; 157:157-66.

MRC. Five-year assessments of controlled trials of ambulatory treatment, debridement and anterior spinal fusion in the management of tuberculosis of the spine: studies in Bulawayo (Rhodesia) and in Hong Kong. J Bone Joint Surg [Br] 1978; 60-B:163-77.

MRC. A ten-year assessment of a controlled trial comparing debridement and anterior spinal fusion in the management of tuberculosis of the spine in patients on standard chemotherapy in Hong Kong. $J$ Bone Joint Surg [Br] 1982; 64-B:393-8.

MRC. A 10-year assessment of controlled trials of in-patient and outpatient treatment and of plaster-of-Paris jackets for tuberculosis of the spine in children on standard chemotherapy: studies in Mason and Pusan, Korea. J Bone Joint Surg /Br] 1985; 67-B:103-10.

MRC. A controlled trial of six-month and nine-month regimens of chemotherapy in patients undergoing radical surgery for tuberculosis of the spine in Hong Kong. Tubercle 1986; 67:243-59.

MRC. Controlled trial of short-course regimens of chemotherapy in the ambulatory treatment of spinal tuberculosis: results at three years of a study in Korea. J Bone Joint Surg /Br/ 1993; 75-B:240-8.

Naim-Ur-Rahman. Atypical forms of spinal tuberculosis. J Bone Joint Surg $[\mathrm{Br}]$ 1980; 62-B:162-5.

Nichols EH. Tuberculosis of bones and joints. Trans Am Orthop Assn 1898; 11:389-91.

Paushter DM, Modic MT, Masaryk TJ. Magnetic resonance imaging of the spine: applications and limitations. Radiol Clin North Am 1985; 23:551-62.

Rao BD, Rao KS, Subramanian MV, Reddy MV. Granulomatous lesions of the spinal epidural space. Neurol India 1965; 13:89-92.

Seddon HJ. Pott's paraplegia: prognosis and treatment. Br J Surg 1935; 22:769-99.

Smith AS, Weinstein MA, Mizushima A, et al. MR imaging characteristics of tuberculous spondylitis vs vertebral osteomyelitis. AJNR 1989; 10:619-25.

Watson JM. Tuberculosis in Britain today (Editorial). BMJ 1993; 306:221-2.

Weaver P, Lifeso RM. The radiological diagnosis of tuberculosis of the adult spine. Skeletal Radiol 1984; 12:178-86. 